\title{
Photoelastic tomography as hybrid mechanics
}

\author{
H. Aben ${ }^{a}$, L. Ainola, A. Errapart \\ Institute of Cybernetics, Tallinn University of Technology, 21 Akadeemia tee, 12618 Tallinn, \\ Estonia
}

\begin{abstract}
Photoelastic tomography is a non-destructive method of 3D stress analysis. It permits determination of normal stress distribution in an arbitrary section of a 3D test object. In case of axial symmetry also the shear stress distribution can be determined directly from the measurement data. To determine also the other stress components one can use equations of the theory of elasticity. Such a combined application of experimental measurements and numerical handling of the equations of the theory of elasticity is named hybrid mechanics. It is shown that if stresses are due to external loads, the hybrid mechanics algorithm is based on the equations of equilibrium and compatibility. In the case of the measurement of the residual stress in glass the compatibility equation can not be applied. In this case a new relationship of axisymmetric thermoelasticity, the generalized sum rule can be applied.
\end{abstract}

\section{Classical tomography}

Tomography is a powerfull method for the analysis of the internal structure of different objects, from human bodies to parts of atomic reactors [1]. In tomography, some radiation (X-rays, protons, acoustic waves, light, etc.) is passed through a section of the object in many directions, and properties of the radiation after it has passed the object (intensity, phase, deflection, etc.) are measured on many rays (Figure 1). Experimental data $g\left(l, \theta^{*}\right)$ for different values of the angle $\theta^{*}$ are called projections.

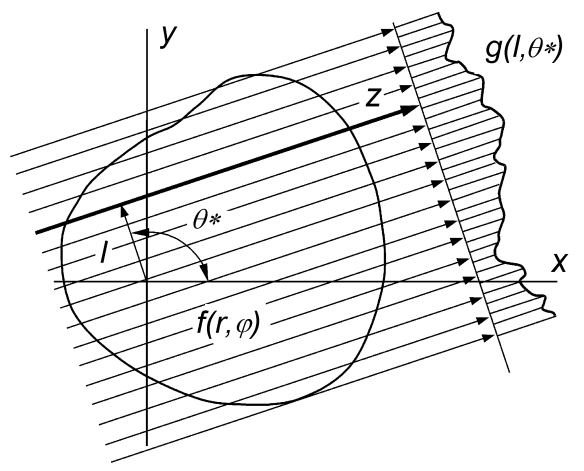

Fig. 1. Scheme of tomographic measurements.

a e-mail : aben@cs.ioc.ee 
If $f(r, \varphi)$ is the function that determines the distribution of a certain parameter of the field, the experimental data for a real pair $l, \theta^{*}$ can be expressed by the Radon transform of the field,

$$
g\left(l, \theta^{*}\right)=\int_{-\infty}^{\infty} f\left[r\left(l, \theta^{*}, z\right), \varphi\left(l, \theta^{*}, z\right)\right] \mathrm{d} z .
$$

When projections for many values of $\theta^{*}$ have been recorded, the function $f(r, \varphi)$ is determined from the Radon inversion

$$
f(r, \varphi)=\frac{1}{2 \pi^{2}} \int_{0}^{\pi} \mathrm{d} \theta^{\prime} \int_{-\infty}^{\infty} \frac{\partial g\left(l, \theta^{*}\right)}{\partial l} \frac{\mathrm{d} l}{r \cos \left(\theta^{*}-\varphi\right)-l} .
$$

Many numerical algorithms for solving Eq. (2) have been elaborated [1].

The question arises whether it is possible to determine tomographically also the stress fields in 3D objects. This problem is not trivial, for the following reason. Classical tomography considers only determination of scalar fields, i.e., every point of the field is characterized by a single number (the coefficient of attenuation of the X-rays, the acoustical or optical index of refraction, etc.). Since stress is a tensor, in stress field tomography every point of the field is characterized by six numbers. Thus the problem is much more complicated in principle. Let us mention that while many books are devoted to scalar field tomography, there is only a single book, written by Sharafutdinov [2], devoted to mathematical problems of the tensor field tomography.

\section{Photoelastic tomography}

\subsection{Linear approximation in integrated photoelasticity}

Let us assume that in two parallel sections $z=\mathrm{z}_{0}$ and $z=z_{0}+\Delta z$ of an arbitrary 3D specimen tomographic photoelastic measurements have been carried out and the integrals $V_{1}$ and $V_{2}$ have been measured for many azimuths $\beta$ (Figure 2):

$$
\begin{gathered}
V_{1}=\Delta \cos 2 \varphi=C \int\left(\sigma_{x^{\prime}}-\sigma_{z^{\prime}}\right) \mathrm{d} y^{\prime}, \\
V_{2}=\Delta \sin 2 \varphi=2 C \int \tau_{x^{\prime} z^{\prime}} \mathrm{d} y^{\prime} .
\end{gathered}
$$

It is assumed that for tomographic measurements the specimen is rotated around the $z$ axis. Equations (3) and (4) are valid if birefringence is weak or rotation of the principal stress axes is small $[3,4]$.

Sharafutdinov suggested the following method for the measurement of the distribution of the axial stress $\sigma_{z}$ [2]. Besides the measurement of the functions $V_{1}$ and $V_{2}$, the value of the axial stress $\sigma_{z}$ is to be measured on the boundary of the cross-section. Applying to the functions $V_{1}$ and $V_{2}$ the transverse ray transformation [2], the $\sigma_{z}$ field is determined from the boundary value problem for the Poisson equation. Sharafutdinov has shown that the solution of this tomographic problem is unique and that only distribution of $\sigma_{z}$ can be determined in this way.

The drawback of this method is that in addition to tomographic photoelastic measurements the boundary values of $\sigma_{z}$ must be measured. That is possible only in the case when boundary of the cross-section is described by a convex curve. Besides, the transverse ray transformation is rather complicated. The tomographic algorithm of Sharafutdinov has not been applied in practice, although it is important from the point of view of the theory of photoelastic tomography. 


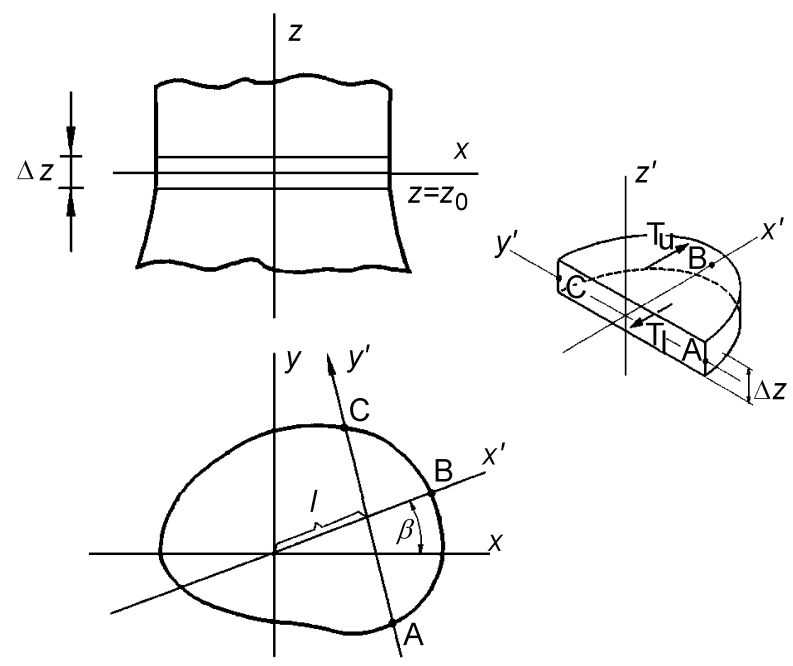

Fig. 2. Illustration explaining tomographic measurement scheme.

Let us mention that Schupp [5] has developed a method for 3D stress field tomography based on interferometric measurement of the absolute optical retardation. Such measurements are carried out by rotating the test object around three mutually perpendicular axes $x, y$, and $\mathrm{z}$. The author also limits himself to linear approximation. The method proposed is mathematically correct and has been proved also with numerical and physical experiments. Unfortunately, the author had to confess that he was able to obtain only qualitative results concerning the stress field. We have to conclude that to base photoelastic tomography on the interferometric measurement of the absolute optical retardations is very complicated.

\subsection{The method of decomposition}

Since Radon inversion for the tensor field does not exist, the problem of stress field tomography can be solved if we can reduce it to a problem of scalar field tomography for a single component of the stress tensor. That can be done in the following way [4,6]. Let us assume that photoelastic tomographic measurements have been carried out on two parallel sections, a distance $\Delta z$ apart from each other, rotating the specimen around the $z$ axis (Figure 2). The values of the functions $V_{1}$ and $V_{2}$ in the auxiliary section we denote $V^{\prime}{ }_{1}$ and $V_{2}^{\prime}$. Considering the equilibrium of the three-dimensional segment $A B C$ in the direction of the $x^{\prime}$ axis (Figure 2), we may write

$$
\Delta z \int_{A}^{C} \sigma_{x^{\prime}} \mathrm{d} y^{\prime}=T_{u}-T_{l},
$$

where $T_{u}$ and $T_{l}$ are the shear forces on the upper and lower surfaces of the segment, respectively:

$$
T_{u}=\frac{1}{2 C} \int_{l}^{B} V_{2}^{\prime} \mathrm{d} x^{\prime}, \quad T_{l}=\frac{1}{2 C} \int_{l}^{B} V_{2} \mathrm{~d} x^{\prime} .
$$

Taking into consideration the relationships (5) and (6), Eq. (3) reveals

$$
\int_{A}^{C} \sigma_{z} \mathrm{~d} y^{\prime}=\frac{1}{2 C \Delta z}\left(\int_{l}^{B} V_{2}^{\prime} \mathrm{d} x^{\prime}-\int_{l}^{B} V_{2} \mathrm{~d} x^{\prime}\right)-\frac{V_{1}}{C} .
$$


Since tomographic photoelastic measurement data can be obtained for all the light rays $y^{\prime}$, Eq. (7) expresses the Radon transform of the field of the stress $\sigma_{z}$. Thus we have reduced a problem of tensor field tomography to a problem of scalar field tomography for a single stress component $\sigma_{z}$. The field of $\sigma_{z}$ can be determined using any of the well-known Radon inversion techniques [1]. Rotating the specimen by tomographic measurements around the axes $x$ and $y$, fields of $\sigma_{x}$ and $\sigma_{y}$ can also been determined.

In case of an axisymmetric stress field, the problem is reduced to a problem of one-dimensional tomography [7]. In this case the distribution of $\sigma_{z}$ is determined from Eq. (7) with Abel inversion [8]. The shear stress $\tau_{x^{\prime} z^{\prime}}$ can be expressed as

$$
\tau_{x^{\prime} z^{\prime}}=\tau_{r z} \cos \beta
$$

where $\tau_{r z}$ is shear stress in cylindrical coordinates. Inserting (8) into (4), a modified algorithm of Abel inversion can be elaborated for the calculation of $\tau_{r z}[8]$.

\section{Algorithms of hybrid mechanics}

\subsection{Stresses due to external loads}

The first hybrid mechanics algorithm for complete determination of the axisymmetric stress field with photoelastic tomography was elaborated by Doyle and Danyluk $[9,10]$. We consider the same problem in a somewhat different way.

Let us express the stress components as power series:

$$
\sigma_{r}=\sum_{k=0}^{m} a_{2 k} \rho^{2 k}, \quad \sigma_{\theta}=\sum_{k=0}^{m} b_{2 k} \rho^{2 k}, \quad \sigma_{z}=\sum_{k=0}^{m} c_{2 k} \rho^{2 k}, \quad \tau_{r z}=\sum_{k=1} d_{2 k-1} \rho^{2 k-1},
$$

where $a_{2 k}, b_{2 k}, c_{2 k}$ and $d_{2 k-1}$ are the coefficients to be determined and $\rho$ is dimensionless radius.

Introducing expression for $\sigma_{z}$ into Eq. (7) we have

$$
\sum_{k=0}^{m} c_{2 k} G_{2 k}=F(x)
$$

where

$$
G_{0}=\sqrt{1-\xi^{2}}, \quad G_{2 k}=\frac{1}{2 k+1}\left(G_{0}+2 k \xi^{2} G_{2 k-2}\right)
$$

and $\xi=x / R$.

Since according to Eq. (7) $F(x)$ has been determined experimentally, formula (10) reveals a system of algebraic equations from which the coefficients $c_{2 k}$ can be determined. Thus, distribution of the axial stress $\sigma_{z}$ has been determined.

Inserting expressions for $\tau_{x^{\prime} z^{\prime}}(8)$ into Eq. (4) reveals after integration

$$
\frac{\Delta \sin 2 \varphi}{4 C R}=\sum_{k=1}^{m} d_{2 k-1} T_{2 k-1}, \quad T_{2 k-1}=\xi G_{2 k-2} .
$$

From the system of equations (12) the shear stress coefficients $d_{2 k-1}$ can be calculated. 
The axial stress $\sigma_{z}$ and the shear stress $\tau_{r z}$ are the only stress components which can be determined directly from measurement data. However, in hybrid mechanics one can use equations of the theory of elasticity. If expansions (9) are introduced into the compatibility equation

$$
\frac{\partial}{\partial \rho}\left[\sigma_{\theta}-\mu\left(\sigma_{r}+\sigma_{z}\right)\right]-(1+\mu) \frac{\sigma_{r}-\sigma_{\theta}}{\rho}=0,
$$

and into the equilibrium equation

$$
\frac{\partial \sigma_{r}}{\partial \rho}+\frac{\sigma_{r}-\sigma_{\theta}}{\rho}+\frac{\partial \tau_{r z}}{\partial \zeta}=0, \quad \zeta=\frac{z}{2\left(R+R^{\prime}\right)},
$$

we obtain after some transformations

$$
\begin{aligned}
& a_{2 k}=\frac{2 k \mu}{(2 k+1)^{2}-1} c_{2 k}-\frac{2 k+1+\mu}{(2 k+1)^{2}-1} d_{2 k-1}^{\prime \prime}, \\
& b_{2 k}=\frac{2 k \mu}{(2 k+1)^{2}-1} c_{2 k}-\frac{1+(2 k+1) \mu}{(2 k+1)^{2}-1} d_{2 k-1}^{\prime \prime},
\end{aligned}
$$

where

$$
d_{2 k-1}^{\prime \prime}=\frac{1}{\delta \zeta}\left[d_{2 k-1}^{\prime}\left(\frac{R}{R^{\prime}}\right)^{2 k-1}-d_{2 k-1}\right]
$$

Thus, stresses due to external loads are completely determined.

Above $R$ denotes the radius of the main section at $z=z_{0}$, and $R^{\prime}$ is radius of the auxiliary section at $z=z_{0}+\Delta z$.

\subsection{The case of residual stresses in glass}

In the case of axial symmetry the compatibility equation written through deformations is

$$
\frac{\partial \varepsilon_{\theta}}{\partial \rho}-\frac{\varepsilon_{r}-\varepsilon_{\theta}}{\rho}=0
$$

The compatibility equation (13) was obtained introducing into Eq. (18) the Hooke's law

$$
\begin{gathered}
\varepsilon_{r}=\frac{1}{E}\left[\sigma_{r}-\mu\left(\sigma_{\theta}+\sigma_{z}\right)\right]+\alpha T, \\
\varepsilon_{\theta}=\frac{1}{E}\left[\sigma_{\theta}-\mu\left(\sigma_{z}+\sigma_{r}\right)\right]+\alpha T, \\
\varepsilon_{z}=\frac{1}{E}\left[\sigma_{z}-\mu\left(\sigma_{r}+\sigma_{\theta}\right)\right]+\alpha T,
\end{gathered}
$$

where $\alpha$ is the thermal expansion coefficient and $T$ is temperature.

In the case of external loads $T$ is either absent or known. In Eq. (13) we have omitted $T$. 
In the case of residual stresses in glass, in Eqs. (19) to (21) the term $\alpha T$ must be included. Since residual stresses in glass have thermal origin, the residual stresses can be considered as being caused by a fictitious temperature field $T[11,12]$. Unfortunately, this temperature field is not known. Therefore, the compatibility equation cannot be used when investigating residual stresses in glass and one has to look for other analytical relationships between the stress components.

Thermal stresses in an axisymmetric body can be expressed as [13]

$$
\begin{gathered}
\sigma_{r}=2 G\left(\frac{\partial^{2} F}{\partial r^{2}}-\Delta F\right)+\frac{2 G}{1-2 \mu} \frac{\partial}{\partial z}\left(\mu \Delta L-\frac{\partial^{2} L}{\partial r^{2}}\right), \\
\sigma_{\theta}=2 G\left(\frac{1}{r} \frac{\partial F}{\partial r}-\Delta F\right)+\frac{2 G}{1-2 \mu} \frac{\partial}{\partial z}\left(\mu \Delta L-\frac{1}{r} \frac{\partial L}{\partial r}\right), \\
\sigma_{z}=2 G\left(\frac{\partial^{2} F}{\partial z^{2}}-\Delta F\right)+\frac{2 G}{1-2 \mu} \frac{\partial}{\partial z}\left[(2-\mu) \Delta L-\frac{\partial^{2} L}{\partial z^{2}}\right], \\
\tau_{r z}=2 G \frac{\partial^{2} F}{\partial r \partial z}+\frac{2 G}{1-2 \mu} \frac{\partial}{\partial r}\left[(1-\mu) \Delta L-\frac{\partial^{2} L}{\partial z^{2}}\right],
\end{gathered}
$$

where $F$ is stress function and $L$ Love's displacement function,

$$
\Delta F=\frac{1+\mu}{1-\mu} \alpha T, \quad \Delta \Delta L=0, \quad G=\frac{E}{2(1+\mu)}, \quad \Delta \frac{\partial^{2}}{\partial r^{2}}+\frac{1}{r} \frac{\partial}{\partial r}+\frac{\partial^{2}}{\partial z^{2}} .
$$

Let us assume that a long cylinder or tube is manufactured by solidifying it in an axisymmetric temperature field without gradient in the axial direction. In this case the thermal (and residual) stresses are the same in all cross-sections of the cylinder, except the parts near the ends of the latter. Now from Eqs. (22) to (24) follows the classical sum rule

$$
\sigma_{r}+\sigma_{\theta}=\sigma_{z}
$$

The classical sum rule (27) was in a somewhat different way first derived by O'Rourke [14].

Inserting expansions (9) into the equilibrium equation (14) and into the sum rule (27), we obtain

$$
a_{2 k}=\frac{1}{2(k+1)} c_{2 k}, \quad b_{2 k}=\frac{2 k+1}{2(k+1)} c_{2 k} .
$$

Thus, in the absence of the stress gradient in axial direction, all the residual stress components can be determined since $c_{2 k}$ have been determined experimentally.

This method has been widely used for residual stress measurement in glass cylinders, axisymmetric fibers and fiber preforms [4]

In the general case, in axisymmetric glass articles stress gradient in axial direction cannot be ignored. Let us try to derive from Eqs. (22) to (25) a relationship between stress components for that case.

If stress gradient in axial direction is present but smooth, we may write

$$
\frac{\partial^{2} F}{\partial z^{2}}=\frac{\partial^{2} L}{\partial z^{2}}=0
$$

Now from Eqs. (22) to (25) follows 


$$
\sigma_{r}+\sigma_{\theta}=\sigma_{z}+\frac{2 G}{1-2 \mu} \frac{\partial}{\partial z}[3(\mu-1) \Delta L]
$$

Differentiating (25) relative to $z$ and integrating along $r$ reveals

$$
\int_{0}^{r} \frac{\partial \tau_{r z}}{\partial z} \mathrm{~d} r=\frac{2 G}{1-2 \mu} \frac{\partial}{\partial z}[(1-\mu) \Delta L]+C(z),
$$

where $C(z)$ is the integration constant.

From (30) and (31) follows

$$
\sigma_{r}+\sigma_{\theta}=\sigma_{z}-3 \int_{0}^{r} \frac{\partial \tau_{r z}}{\partial z} \mathrm{~d} r+C(z) .
$$

The last relationship is named the generalized sum rule in first approximation. By handling Eqs. (22) to (25) asymptotically, the second approximations of the generalized sum rule can be obtained:

$$
\sigma_{r}+\sigma_{\theta}=\sigma_{z}-2 \int_{0}^{r} \frac{\partial \tau_{r z}}{\partial z} \mathrm{~d} r+C_{1}(z) .
$$

By using the photoelastic measurement data, equilibrium equation (13) and the generalized sum rule (33), all components of the residual stress in an axisymmetric glass article can be determined.

As an example, Figure 3 shows geometry of a wine glass and fringe pattern in the area of the lower part of the stem. Using the hybrid algorithm described above, all the stress components were determined in the section B - B (Figure 4).

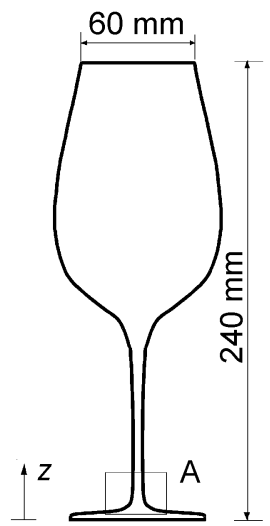

(a)

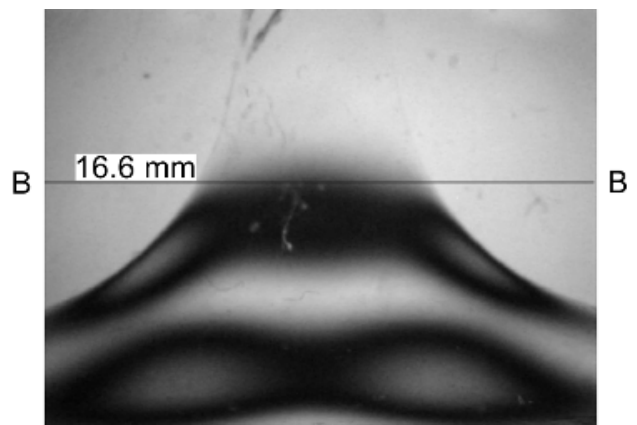

(b)

Fig. 3. Geometry of the wine glass (a) and fringe pattern in the area A (b). 


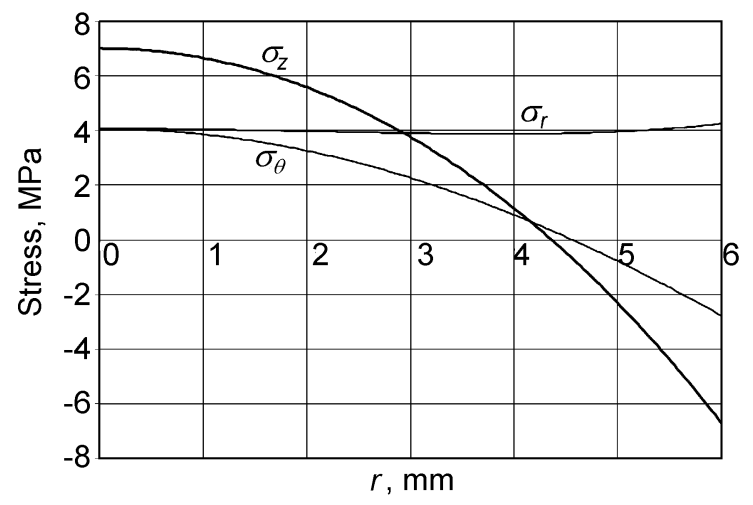

Fig. 4. Normal stress components in section B - B.

\section{Conclusions}

It has been shown that using equations of the theory of elasticity, hybrid algorithms can be constructed for complete determination of axisymmetric stress fields in photoelastic tomography. Determination of the residual stress in glass needs application of a different algorithm as compared with the case of external loads.

\section{Acknowledgement}

Support of the Estonian Science Foundation (grant No. 7840) is gratefully acknowledged.

\section{References}

1. C. T. Herman, Image Reconstruction from Projections (Academic Press, New York, 1980)

2. V. A. Sharafutdinov, Integral Geometry of Tensor Fields (VSP, Utrecht, 1994)

3. H. K. Aben, J. I. Josepson, K.-J. Kell, Opt. Lasers Eng. 11, 145 (1989)

4. H. Aben, C. Guillemet, Photoelasticity of Glass (Springer, Berlin, 1993)

5. D. Schupp, Tech. Mess. 66, 54 (1999)

6. H. Aben, A. Errapart, L. Ainola, J. Anton, Opt. Eng. 44, 093601-1 (2005)

7. C. J. Dash, Appl. Opt. 31, 1146 (1992)

8. H. Aben, L. Ainola, A. Errapart, Inverse Problems in Sci. Eng., 18 (2010)

9. J. F. Doyle, H. T. Danyluk, Exp. Mech. 18, 215 (1978)

10. J. F. Doyle, J. Appl. Mech. 47, 431 (1980)

11. G. M. Bartenev, The Structure and Mechanical Properties of Inorganic Glasses (WoltersNordhoff, Groningen, 1970)

12. R. Gardon, Glass Science and Technology, Vol. 5, 146 (Academic Press, New York, 1980)

13. E. Melan, H. Parkus, Wärmespannungen infolge stationärer Temperaturfelder (Springer, Wien, 1953)

14. R. C. O’Rourke, J. Appl. Phys, 22, 872 (1951) 